Commun. Korean Math. Soc. 27 (2012), No. 3, pp. 455-464

http://dx.doi.org/10.4134/CKMS.2012.27.3.455

\title{
ON THE RELATIVE ZETA FUNCTION IN FUNCTION FIELDS
}

\author{
DAISUKe Shiomi
}

\begin{abstract}
In the previous paper [8], the author gave a determinant formula of relative zeta function for cyclotomic function fields. Our purpose of this paper is to extend this result for more general function fields. As an application of our formula, we will give determinant formulas of class numbers for constant field extensions.
\end{abstract}

\section{Introduction}

Let $p$ be a prime. In 1955, Carlitz and Olson [2] provided an expression of the relative class number of $p$-th cyclotomic field in terms of a certain classical determinant, which is known as the Maillet determinant. Many authors have extended this result (cf. [5], [9]). In particular, Girstmair [3] gave a generalization to an imaginary abelian field.

In the function field case, several authors also gave determinant formulas for class numbers. Let $\mathbb{F}_{q}$ be the field with $q$ elements. Let $k=\mathbb{F}_{q}(T)$ be the rational function field over $\mathbb{F}_{q}$, and $A=\mathbb{F}_{q}[T]$ the associated polynomial ring. Let $m \in A$ be a monic polynomial. We denote by $\Lambda_{m}$ the set of all $m$-torsion points of the Carlitz module (see Section 2). Let $K_{m}=k\left(\Lambda_{m}\right)$. The function field $K_{m}$ is called the $m$-th cyclotomic function field. This function field has remarkably similar properties of those of cyclotomic field over $\mathbb{Q}$.

We assume that $m$ is irreducible. In 1990s, Rosen gave a determinant formula for the relative class number of $K_{m}$ (cf. [6]), which is regarded as an analogue of the formula of Carlitz and Olson. Recently, Ahn, Choi, and Jung generalized the Rosen's formula to any subfield of cyclotomic function fields with arbitrary conductor (cf. [1]). On the other hand, in the previous paper [8], the author gave a determinant formula for a relative zeta function of cyclotomic function field.

Our goal of this paper is to generalize these formulas. Let $M$ be a finite extension over $k$ which is contained in some cyclotomic function fields. Put $\zeta^{(-)}(s, M)=\zeta(s, M) / \zeta\left(s, M^{+}\right)$, where $\zeta(s, M), \zeta\left(s, M^{+}\right)$are zeta functions of

Received February 2, 2011.

2010 Mathematics Subject Classification. 11M38, 11R58.

Key words and phrases. zeta functions, class numbers, function fields.

(C)2012 The Korean Mathematical Society 
$M$, and the maximal real subfield of $M$, respectively. We shall call $\zeta^{(-)}(s, M)$ the relative zeta function of $M$. First we will show that $\zeta^{(-)}(s, M)$ can be expressed by the polynomial $Z_{M}^{(-)}(u)$ of integral coefficients (see Section 2). The main result of this paper is to construct a matrix $E_{M}^{(\lambda)}(u)$, and give the following determinant formula of relative zeta function:

$$
\prod_{\substack{\lambda \in T_{M} \\ \lambda \neq 1}} \operatorname{det} E_{M}^{(\lambda)}(u)=Z_{M}^{(-)}(u) J_{M}(u)
$$

(see Theorem 3.1), where the matrix $E_{M}^{(\lambda)}(u)$ and the polynomial $J_{M}(u)$ are calculated automatically when once $M$ is given (see Section 3). Considering the case $M=K_{m}$, the above formula leads the determinant formula which the author gave in [8].

As an application of our formula, we will give a class number formula of $M_{n}$, where $M_{n}$ is the constant field extension over $M$ of degree $n$ (see Theorem 3.2). We consider the case $n=1$. Then our class number formula derives the determinant formula of Ahn-Choi-Jung (cf. Theorem 3.2 in [1]).

\section{Preparations}

In this section, we will provide definitions and basic properties of cyclotomic function fields, and zeta functions. For details, see [4], [7], [10].

\subsection{Cyclotomic function field and Dirichlet characters}

Let $\bar{k}$ be an algebraic closure of $k$. For $x \in \bar{k}$ and $m \in A$, we define the following action:

$$
m * x=m(\varphi+\mu)(x),
$$

where $\varphi, \mu$ are $\mathbb{F}_{q}$-linear isomorphisms of $\bar{k}$ defined by $\varphi: x \mapsto x^{q}$, and $\mu$ : $x \mapsto T x$, respectively. By this action, $\bar{k}$ becomes $A$-module, which is called the Carlitz module. For a monic polynomial $m \in A$, we denote by $\Lambda_{m}$ the set of all $x \in \bar{k}$ satisfying $m * x=0$. Let $K_{m}=k\left(\Lambda_{m}\right)$. We call $K_{m}$ the $m$-th cyclotomic function field. Then it is well-known that $K_{m} / k$ is a Galois extension, and its Galois group is isomorphic to $G_{m}$, where $G_{m}$ is the unit group of $A / m A$. We regard $\mathbb{F}_{q}^{\times} \subseteq G_{m}$, and denote by $K_{m}^{+}$the intermediate field of $K_{m} / k$ corresponding to $\mathbb{F}_{q}^{\times}$. Let $P_{\infty}$ be the prime of $k$ with the valuation ord ${ }_{\infty}$ satisfying $\operatorname{ord}_{\infty}(1 / T)=1$. We denote the associated completion of $k$ by $k_{\infty}$. Then $K_{m}^{+}=k_{\infty} \cap K_{m}$.

Next we put

$$
\widetilde{K}=\bigcup_{m: \text { monic }} K_{m}
$$

where $m$ runs through all monic polynomials of $A$. Let $M \subseteq \widetilde{K}$ be a finite extension over $k$. We define the conductor of $M$ as the monic polynomial $m \in A$ of the smallest degree satisfying $M \subseteq K_{m}$. We call $M$ real if $M \subseteq k_{\infty}$. 
Otherwise, we call $M$ imaginary. Put $M^{+}=M \cap k_{\infty}$. Then $M^{+}$is the maximal real subfield of $M$.

Lemma 2.1. Let $M \subseteq \widetilde{K}$ be a finite extension of $k$. Then $P_{\infty}$ splits completely in $M^{+} / k$, and each prime of $M^{+}$above $P_{\infty}$ is totally ramified in $M / M^{+}$.

Proof. This follows from Theorem 12.14 in [7].

Next we review basic facts about Dirichlet characters. For a monic polynomial $m \in A$, let $X_{m}$ be the group of all primitive Dirichlet characters of $G_{m}$. We denote by $X$ the group of all primitive Dirichlet characters (i.e., $X=\cup_{m} X_{m}$ ). Then, by the same argument as in the case of number field, we have a oneto-one correspondence between finite subgroups of $X$ and intermediate fields of $\widetilde{K} / k$ of finite degree (cf. Chapter 3 in [10]). In particular, we see that $K_{m}$ corresponds to $X_{m}$. Let $M \subseteq \widetilde{K}$ be a finite extension of $k$. We denote by $X_{M}$ the finite subgroup of $X$ corresponding to $M$. Put

$$
X_{M}^{+}=\left\{\chi \in X_{M} \mid \chi(a)=1 \text { for all } a \in \mathbb{F}_{q}^{\times}\right\} .
$$

We see that $M^{+}$corresponds to $X_{M}^{+}$. We will use the next theorem in the later section.

Theorem 2.1 (cf. [10], Theorem 3.7). Let $M \subseteq \widetilde{K}$ be a finite extension of $k$. For an irreducible monic polynomial $Q \in A$, put

$$
Y_{M, Q}=\left\{\chi \in X_{M} \mid \chi(Q) \neq 0\right\}, Z_{M, Q}=\left\{\chi \in X_{M} \mid \chi(Q)=1\right\} .
$$

Then $Y_{M, Q} / Z_{M, Q}$ is a cyclic group of order $f_{Q}$, and the order of $Z_{M, Q}$ is equal to $g_{Q}$, where $f_{Q}, g_{Q}$ are the relative degree of $Q$ in $M / k$, and the number of primes of $M$ above $Q$, respectively.

\subsection{Zeta functions and $L$-functions}

For a global function field $M$ over $\mathbb{F}_{q}$, we define the zeta function by

$$
\zeta(s, M)=\prod_{\mathcal{P}: \text { prime }}\left(1-\frac{1}{\mathcal{N} \mathcal{P}^{s}}\right)^{-1},
$$

where $\mathcal{P}$ runs through all primes of $M$, and $\mathcal{N} \mathcal{P}$ is the number of elements of the residue class field of $\mathcal{P}$. We can easily check that $\zeta(s, M)$ converges absolutely for $\operatorname{Re}(s)>1$.

Theorem 2.2 (cf. [7] Theorem 5.9). In the above notations, there is a polynomial $Z_{M}(u)$ with integral coefficients such that

$$
\zeta(s, M)=\frac{Z_{M}\left(q^{-s}\right)}{\left(1-q^{1-s}\right)\left(1-q^{-s}\right)} .
$$

Moreover, $Z_{M}(0)=1, Z_{M}(1)=h_{M}$, where $h_{M}$ is the class number of $M$.

We denote by $\mathbb{F}_{q^{n}}$ the extension over $\mathbb{F}_{q}$ of degree $n$. Put $M_{n}=M \mathbb{F}_{q^{n}}$, and let $h_{M, n}$ be the class number of $M_{n}$. Notice that $h_{M, 1}=h_{M}$. 
Proposition 2.1. In the above notations, we have

$$
\prod_{\omega^{n}=1} Z_{M}(\omega)=h_{M, n}
$$

where $\omega$ runs through $n$-th roots of unity.

Proof. This follows from Proposition 8.16 in [7].

Next we will review basic facts about $L$-functions. For a character $\chi \in X$, we define the $L$-function by

$$
L(s, \chi)=\prod_{P}\left(1-\frac{\chi(P)}{\mathcal{N} P^{s}}\right)^{-1},
$$

where $P$ runs through all irreducible monic polynomials of $A$. Let $m$ be the conductor of $\chi$. For $\alpha \in G_{m}$, there is the unique element $r_{\alpha} \in A$ satisfying

$$
\begin{aligned}
& r_{\alpha}=a_{n} T^{n}+a_{n-1} T^{n-1}+\cdots+a_{0} \quad\left(n=\operatorname{deg} r_{\alpha}<\operatorname{deg} m\right), \\
& r_{\alpha} \equiv \alpha \bmod m .
\end{aligned}
$$

Then we define functions Deg and L over $G_{m}$ by

$$
\operatorname{Deg}(\alpha)=n, \quad \mathrm{~L}(\alpha)=a_{n} \in \mathbb{F}_{q}^{\times} .
$$

Put

$$
G_{m}^{(1)}=\left\{\alpha \in G_{m} \mid \mathrm{L}(\alpha)=1\right\} .
$$

Then $L(s, \chi)$ can be expressed as follows:

$$
L(s, \chi)= \begin{cases}1 /\left(1-q^{1-s}\right) & \text { if } \chi=\chi_{0}, \\ \sum_{\alpha \in G_{m}^{(1)}} \chi(\alpha) q^{-s \operatorname{Deg}(\alpha)} & \text { otherwise, }\end{cases}
$$

where $\chi_{0}$ is the trivial character (cf. Proposition 4.3 in [7]). Let $M \subseteq \widetilde{K}$ be a finite extension of $k$. By the same arguments as in the case of number fields, we have

$$
\prod_{\mathcal{P}: \text { finite }}\left(1-\frac{1}{\mathcal{N} \mathcal{P}^{s}}\right)^{-1}=\prod_{\chi \in X_{M}} L(s, \chi),
$$

where $\mathcal{P}$ runs over all primes of $M$ not above $P_{\infty}$ (cf. Theorem 4.3 in [10]). By Lemma 2.1 and the equation (2), we have

$$
\begin{aligned}
\zeta(s, M) & =\left\{\prod_{\chi \in X_{M}} L(s, \chi)\right\}\left(1-q^{-s}\right)^{-\left[M^{+}: k\right]}, \\
\zeta\left(s, M^{+}\right) & =\left\{\prod_{\chi \in X_{M}^{+}} L(s, \chi)\right\}\left(1-q^{-s}\right)^{-\left[M^{+}: k\right]} .
\end{aligned}
$$


We define the relative zeta function of $M$ by $\zeta^{(-)}(s, M)=\zeta(s, M) / \zeta\left(s, M^{+}\right)$. Then we have

$$
\zeta^{(-)}(s, M)=\prod_{\chi \in X_{M}^{-}} L(s, \chi)
$$

where $X_{M}^{-}=X_{M} \backslash X_{M}^{+}$. We put $Z_{M}^{(-)}(u)=Z_{M}(u) / Z_{M^{+}}(u)$ (see Theorem 2.2). Then we have $\zeta^{(-)}(s, M)=Z_{M}^{(-)}\left(q^{-s}\right)$.

Proposition 2.2. In the above notations, $Z_{M}^{(-)}(u)$ is a polynomial of integral coefficients.

Proof. For a non-trivial character $\chi \in X_{M}$, we saw that $L(s, \chi)$ is a polynomial of $q^{-s}$ with complex coefficients. Hence $Z_{M}^{(-)}(u)$ is a polynomial with complex coefficients. On the other hand, we notice that $Z_{M}(u)$ and $Z_{M^{+}}(u)$ are polynomials of integral coefficients, and $Z_{M}(0)=Z_{M^{+}}(0)=1$. Therefore $Z_{M}^{(-)}(u)$ is a polynomial of integral coefficients.

In the end of this section, we give a class number formula of constant field extension. For a positive integer $n$, put $h_{M, n}^{-}=h_{M, n} / h_{M^{+}, n} \in \mathbb{Q}$. In particular, $h_{M, 1}^{-}$is the relative class number of $M$. From Proposition 2.1, we have the following class number formula:

$$
h_{M, n}^{-}=\prod_{\omega^{n}=1} Z_{M}^{(-)}(\omega) .
$$

By the above formula, we see that $h_{M, n}^{-}$is an algebraic integer. Hence $h_{M, n}^{-}$is an integer.

\section{Determinant formulas for zeta functions}

In the previous section, we defined the relative zeta function $\zeta^{(-)}(s, M)$ for a finite extension $M \subseteq \widetilde{K}$ over $k$, and we saw that $\zeta^{(-)}(s, M)$ is expressed by the polynomial $Z_{M}^{(-)}(u)$. Our goal in this section is to construct a determinant formula for $Z_{M}^{(-)}(u)$. As an application of this formula, we will give determinant formulas for class numbers of constant field extensions over $M$.

First we give some notations. Let $M \subseteq \widetilde{K}$ be a finite extension over $k$ of conductor $m$. Let $H_{M}, H_{M}^{+}$be subgroups of $G_{m}$ corresponding to $M$, and $M^{+}$, respectively. We see that $H_{M}^{+}=H_{M} \mathbb{F}_{q}^{\times}$. Put $N_{M}=[M: k], N_{M}^{+}=\left[M^{+}: k\right]$, and $N_{M}^{-}=\left[M: M^{+}\right]$. Let $\hat{\mathbb{F}}_{q}^{\times}$be the character group of $\mathbb{F}_{q}^{\times}$. We define the subgroup $T_{M}$ of $\hat{\mathbb{F}}_{q}^{\times}$as follows:

$$
T_{M}=\left\{\left.\chi\right|_{\mathbb{F}_{q}^{\times}} \mid \chi \in X_{M}\right\},
$$

where $\left.\chi\right|_{\mathbb{F}_{q}^{\times}}$is the restriction of $\chi$ to $\mathbb{F}_{q}^{\times}$. Then we see that ${ }^{\#} T_{M}=N_{M}^{-}$, where \# $S$ is the number of elements of a set $S$. We notice that

$$
\chi(a)=1 \quad\left(\chi \in T_{M}, a \in I_{M}\right)
$$


where $I_{M}=H_{M} \cap \mathbb{F}_{q}^{\times}$. For each $\lambda \in T_{M}$, fix a $\psi_{\lambda} \in X_{M}$ satisfying $\left.\psi_{\lambda}\right|_{\mathbb{F}_{q}^{\times}}=\lambda$. Then we have the following decomposition:

$$
X_{M}^{-}=\bigcup_{\substack{\lambda \in T_{M} \\ \lambda \neq 1}} \psi_{\lambda} X_{M}^{+}
$$

Let $\left\{\alpha_{1}, \alpha_{2}, \ldots, \alpha_{N_{M}^{+}}\right\}$be a complete system of representatives for $G_{m} / H_{M}^{+}$. For $\lambda \in T_{M}$ and $i, j=1,2, \ldots, N_{M}^{+}$, we put

$$
F_{i j}^{(\lambda)}(u)=\sum_{\beta \in S_{M}} \lambda\left(L\left(\alpha_{i} \alpha_{j}^{-1} \beta\right)^{-1}\right) u^{\operatorname{Deg}\left(\alpha_{i} \alpha_{j}^{-1} \beta\right)},
$$

where $S_{M}$ is a complete system of representatives for $H_{M} / I_{M}$. By using these polynomials, we define the matrix $E_{M}^{(\lambda)}(u)$ by

$$
E_{M}^{(\lambda)}(u)=\left(F_{i j}^{(\lambda)}(u)\right)_{i, j=1,2, \ldots, N_{M}^{+}}
$$

for $\lambda \in T_{M}$. Then we have the following determinant formula of the relative zeta function.

Theorem 3.1. In the above notations, we have

$$
\prod_{\substack{\lambda \in T_{M} \\ \lambda \neq 1}} \operatorname{det} E_{M}^{(\lambda)}(u)=Z_{M}^{(-)}(u) J_{M}(u) .
$$

The polynomial $J_{M}(u)$ is defined by

$$
J_{M}(u)=\prod_{\chi \in X_{M}^{-}} \prod_{Q \mid m}\left(1-\chi(Q) u^{\operatorname{deg} Q}\right),
$$

where $Q$ runs through irreducible monic polynomials dividing $m$.

Remark 3.1. We consider the case $M=K_{m}$. We notice that $T_{K_{m}}=\hat{\mathbb{F}}_{q}^{\times}$, and $S_{K_{m}}=\{1\}$. Hence the above formula derives the determinant formula which the author gave in [8].

Remark 3.2. We see that $F_{i j}^{(\lambda)}(u) \in \mathbb{Z}[\omega][u]$, where $\omega$ is a primitive $N_{M}^{-}$-th root of unity. In particular, each component of $E_{M}^{(\lambda)}(u)$ is a polynomial of integral coefficients if $N_{M}^{-}=\left[M: M^{+}\right]=2$.

Proposition 3.1. In the notations of Theorem 3.1, we have

$$
J_{M}(u)=\prod_{Q \mid m} \frac{\left(1-u^{f_{Q} \operatorname{deg} Q}\right)^{g_{Q}}}{\left(1-u^{f_{Q}^{+} \operatorname{deg} Q}\right)^{g_{Q}^{+}}},
$$

where $f_{Q}, f_{Q}^{+}$are relative degrees of $Q$ in $M / k, M^{+} / k$ respectively, and $g_{Q}$, $g_{Q}^{+}$are numbers of primes of $M, M^{+}$above $Q$, respectively. 
Proof. Let $Q$ be an irreducible monic polynomial dividing $m$. In the notations of Theorem 2.1, we have

$$
\begin{aligned}
\prod_{\chi \in X_{M}}\left(1-\chi(Q) u^{\operatorname{deg} Q}\right) & =\prod_{\chi \in Y_{M, Q}}\left(1-\chi(Q) u^{\operatorname{deg} Q}\right) \\
& =\prod_{\phi \in Y_{M, Q} / Z_{M, Q}} \prod_{\psi \in Z_{M, Q}}\left(1-\phi \psi(Q) u^{\operatorname{deg} Q}\right) \\
& =\left(\prod_{\phi \in Y_{M, Q} / Z_{M, Q}}\left(1-\phi(Q) u^{\operatorname{deg} Q}\right)\right)^{g_{Q}},
\end{aligned}
$$

where $\phi$ runs through a complete system of representative for $Y_{M, Q} / Z_{M, Q}$. Noting that $Y_{M, Q} / Z_{M, Q}$ is a cyclic group of order $f_{Q}$, we have

$$
\prod_{\phi \in Y_{M, Q} / Z_{M, Q}}\left(1-\phi(Q) u^{\operatorname{deg} Q}\right)=\left(1-u^{f_{Q} \operatorname{deg} Q}\right) .
$$

Therefore

$$
\prod_{\chi \in X_{M}}\left(1-\chi(Q) u^{\operatorname{deg} Q}\right)=\left(1-u^{f_{Q} \operatorname{deg} Q}\right)^{g_{Q}} .
$$

By the same argument, we have

$$
\prod_{\chi \in X_{M}^{+}}\left(1-\chi(Q) u^{\operatorname{deg} Q}\right)=\left(1-u^{f_{Q}^{+} \operatorname{deg} Q}\right)^{g_{Q}^{+}} .
$$

Hence we obtain Proposition 3.1.

Remark 3.3. From the above proposition, we see that $J_{M}(u)$ is a polynomial of integral coefficients.

Remark 3.4. We assume that $m$ is a power of an irreducible monic polynomial $Q$. By Proposition 12.7 in [7], we see that $Q$ is totally ramified in $M / k$. Therefore, by Proposition 3.1, we have $J_{M}(u)=1$.

Now we give the proof of Theorem 3.1.

Proof. Let $\chi \in X_{M}^{-}$, and denote by $f_{\chi}$ the conductor of $\chi$. We see that $f_{\chi}$ divides $m$. Put $\tilde{\chi}=\chi \circ \pi_{\chi}$, where $\pi_{\chi}: G_{m} \rightarrow G_{f_{\chi}}$ is the natural homomorphism. Then we have

$$
L(s, \tilde{\chi})=L(s, \chi)\left\{\prod_{Q \mid m}\left(1-\chi(Q) q^{-s \operatorname{deg} Q}\right)\right\} .
$$

By the equation (3), we obtain

$$
\begin{aligned}
\prod_{\chi \in X_{M}^{-}} L(s, \tilde{\chi}) & =\left(\prod_{\chi \in X_{M}^{-}} L(s, \chi)\right) \cdot J_{M}\left(q^{-s}\right) \\
& =Z_{M}^{(-)}\left(q^{-s}\right) J_{M}\left(q^{-s}\right) .
\end{aligned}
$$


On the other hand, from the equation (4), we have

$$
\prod_{\chi \in X_{M}^{-}} L(s, \tilde{\chi})=\prod_{\substack{\lambda \in T_{M} \\ \lambda \neq \lambda_{0}}} \prod_{\chi \in X_{M}^{+}} L\left(s, \tilde{\psi}_{\lambda} \tilde{\chi}\right) .
$$

Next we will show that

$$
\operatorname{det} E_{M}^{(\lambda)}\left(q^{-s}\right)=\prod_{\chi \in X_{M}^{+}} L\left(s, \tilde{\psi}_{\lambda} \tilde{\chi}\right)
$$

for each non-trivial character $\lambda \in T_{M}$. Fix a non-trivial character $\lambda \in T_{M}$ and $s \in \mathbb{C}$. For $\alpha \in G_{m}$, we put

$$
F(\alpha, \lambda)=\sum_{\beta \in T(\alpha)} \tilde{\psi}_{\lambda}(\beta) q^{-s \operatorname{Deg}(\beta)},
$$

where $T(\alpha)=G_{m}^{(1)} \cap \alpha H_{M}^{+}$. We see that $F(*, \lambda)$ is a function over $G_{m} / H_{M}^{+}$. Noting that $\left\{\alpha_{1}, \alpha_{2}, \ldots, \alpha_{N_{M}^{+}}\right\}$is a complete system of representatives for $G_{m} / H_{M}^{+}$, we obtain

$$
\begin{aligned}
L\left(s, \tilde{\chi} \tilde{\psi}_{\lambda}\right) & =\sum_{\alpha \in G_{m}^{(1)}} \tilde{\chi}(\alpha) \tilde{\psi}_{\lambda}(\alpha) q^{-s \operatorname{Deg}(\alpha)} \\
& =\sum_{i=1}^{N_{M}^{+}} \tilde{\chi}\left(\alpha_{i}\right) F\left(\alpha_{i}, \lambda\right)
\end{aligned}
$$

for $\chi \in X_{M}^{+}$. We see that $\left\{\tilde{\chi} \mid \chi \in X_{M}^{+}\right\}$is the character group of $G_{m} / H_{M}^{+}$. Hence we apply the group determinant formula for the group $G_{m} / H_{M}^{+}$and the function $F(*, \lambda)$ (cf. [10] Lemma 5.26), and obtain

$$
\prod_{\chi \in X_{M}^{+}} L\left(s, \tilde{\chi} \tilde{\psi}_{\lambda}\right)=\operatorname{det}\left(F\left(\alpha_{i} \alpha_{j}^{-1}, \lambda\right)\right)_{i, j=1,2, \ldots, N_{M}^{+}} .
$$

Notice that

$$
T(\alpha)=\left\{\frac{\alpha \beta}{L(\alpha \beta)} \mid \beta \in S_{M}\right\}
$$

for $\alpha \in G_{m}$. Since $\tilde{\psi}_{\lambda}(\alpha)=1$ for all $\alpha \in H_{M}$, we have

$$
F\left(\alpha_{i} \alpha_{j}^{-1}, \lambda\right)=\tilde{\psi}_{\lambda}\left(\alpha_{i} \alpha_{j}^{-1}\right) F_{i j}^{(\lambda)}\left(q^{-s}\right) .
$$

This leads the equation (8). By equations (5), (6), and (7), we have

$$
\prod_{\substack{\lambda \in T_{M} \\ \lambda \neq 1}} \operatorname{det} E_{M}^{(\lambda)}\left(q^{-s}\right)=Z_{M}^{(-)}\left(q^{-s}\right) J_{M}\left(q^{-s}\right) .
$$

Putting $u=q^{-s}$, we obtain Theorem 3.1. 
As an application of our formula, we will give determinant formulas for class numbers of constant field extensions over $M$. To see this, we recall that $h_{M, n}^{-}=\prod_{\omega^{n}=1} Z_{M}^{(-)}(\omega)$ for a positive integer $n$. Hence Theorem 3.1 leads the following class number formula.

Theorem 3.2. In the above notations, we have

$$
\prod_{\omega^{n}=1} \prod_{\substack{\lambda \in T_{M} \\ \lambda \neq 1}} \operatorname{det} E_{M}^{(\lambda)}(\omega)=h_{M, n}^{-} R_{M, n},
$$

where $R_{M, n}$ is defined by $R_{M, n}=\prod_{\omega^{n}=1} J_{M}(\omega)$.

Remark 3.5. Recall that $J_{M}(u)=1$ if $m$ is a power of an irreducible polynomial. Hence $R_{M, n}=1$ in this case.

Proposition 3.2. In the notations of Proposition 3.1, we have

$$
R_{M, n}=\left\{\begin{array}{cc}
\prod_{Q \mid m}\left(\frac{f_{Q}}{f_{Q}^{+}}\right)^{g_{Q}\left(f_{Q} \operatorname{deg} Q, n\right)} & \text { if }\left(f_{Q} \operatorname{deg} Q, n\right)=\left(f_{Q}^{+} \operatorname{deg} Q, n\right) \text { and } \\
& g_{Q}=g_{Q}^{+} \text {for every } Q \text { dividing } m, \\
\text { otherwise, }
\end{array}\right.
$$

where $(a, b)$ is the $G C D$ of $a$ and $b$.

Proof. We see that

$$
\prod_{\omega^{a}=1}\left(1-\omega^{b} u^{b}\right)=\left(1-u^{[a, b]}\right)^{(a, b)},
$$

where $[a, b]$ is the LCM of $a$ and $b$ (cf. [7] Lemma 8.14). Therefore, by Proposition 3.1, we have

$$
\prod_{\omega^{n}=1} J_{M}(\omega u)=\prod_{Q \mid m} \frac{\left(1-u^{\left[f_{Q} \operatorname{deg} Q, n\right]}\right)^{g_{Q}\left(f_{Q} \operatorname{deg} Q, n\right)}}{\left(1-u^{\left[f_{Q}^{+} \operatorname{deg} Q, n\right]}\right)_{Q}^{+}\left(f_{Q}^{+} \operatorname{deg} Q, n\right)} .
$$

Fix an irreducible monic polynomial $Q$ dividing $m$. If $\left(f_{Q} \operatorname{deg} Q, n\right)=\left(f_{Q}^{+} \operatorname{deg} Q\right.$, $n$ ) and $g_{Q}=g_{Q}^{+}$, then we apply L'Hôpital's rule and obtain

$$
\begin{aligned}
\left.\frac{\left(1-u^{\left[f_{Q} \operatorname{deg} Q, n\right]}\right)^{g_{Q}\left(f_{Q} \operatorname{deg} Q, n\right)}}{\left(1-u^{\left[f_{Q}^{+} \operatorname{deg} Q, n\right]}\right)^{g_{Q}^{+}\left(f_{Q}^{+} \operatorname{deg} Q, n\right)}}\right|_{u=1} & =\left(\frac{\left[f_{Q} \operatorname{deg} Q, n\right]}{\left[f_{Q}^{+} \operatorname{deg} Q, n\right]}\right)^{g_{Q}\left(f_{Q} \operatorname{deg} Q, n\right)} \\
& =\left(\frac{f_{Q}}{f_{Q}^{+}}\right)^{g_{Q}\left(f_{Q} \operatorname{deg} Q, n\right)}
\end{aligned}
$$

Otherwise,

$$
\left.\frac{\left(1-u^{\left[f_{Q} \operatorname{deg} Q, n\right]}\right)^{g_{Q}\left(f_{Q} \operatorname{deg} Q, n\right)}}{\left(1-u^{\left[f_{Q}^{+} \operatorname{deg} Q, n\right]}\right)^{g_{Q}^{+}\left(f_{Q}^{+} \operatorname{deg} Q, n\right)}}\right|_{u=1}=0 .
$$

This leads Proposition 3.2.

Remark 3.6. By the above proposition, we see that $R_{M, n}$ is an integer. 
Remark 3.7. Considering the case $n=1$ in Theorem 3.2, we have a relative class number formula for $M$. This formula coincides with the determinant formula of Ahn-Choi-Jung (cf. Theorem 3.2 in [1]).

Example 3.1. Let $q=3, m=T^{3}$. Put

$$
H=\left\{1, T+1, T^{2}+2 T+1\right\} \subset G_{m} .
$$

Let $M$ be the intermediate field of $K_{m} / k$ corresponding to $H$. Then $I_{M}=\{1\}$, $S_{M}=H, N_{M}^{-}=2$. We put $T_{M}=\left\{\lambda_{0}, \lambda\right\}$, and

$$
\alpha_{1}=1, \alpha_{2}=T^{2}+1, \alpha_{3}=2 T^{2}+1 \text {. }
$$

Then we have

$$
E_{M}^{(\lambda)}(u)=\left(\begin{array}{ccc}
u^{2}+u+1 & -2 u^{2}-u & u^{2} \\
u^{2} & u^{2}+u+1 & -2 u^{2}-u \\
-2 u^{2}-u & u^{2} & u^{2}+u+1
\end{array}\right), \quad J_{M}(u)=1,
$$

and

$$
E_{M}^{(\lambda)}(1)=\left(\begin{array}{ccc}
3 & -3 & 1 \\
1 & 3 & -3 \\
-3 & 1 & 3
\end{array}\right), \quad E_{M}^{(\lambda)}(-1)=\left(\begin{array}{ccc}
1 & -1 & 1 \\
1 & 1 & -1 \\
-1 & 1 & 1
\end{array}\right)
$$

By applying Theorem 3.1, we have $Z_{M}^{(-)}(u)=\operatorname{det} E_{M}^{(\lambda)}(u)=9 u^{4}+9 u^{3}+$ $6 u^{2}+3 u+1$. By Theorem 3.2, $h_{M, 1}^{-}=\operatorname{det} E_{M}^{(\lambda)}(1)=28$, and $h_{M, 2}^{-}=$ $\operatorname{det} E_{M}^{(\lambda)}(1) \operatorname{det} E_{M}^{(\lambda)}(-1)=112$.

\section{References}

[1] J. Ahn, S. Choi, and H. Jung, Class number formulae in the form of a product of determinants in function fields, J. Aust. Math. Soc. 78 (2005), no. 2, 227-238.

[2] L. Carlitz and F. R. Olson, Maillet's determinant, Proc. Amer. Math. Soc. 6 (1955), $265-269$.

[3] K. Girstmair, The relative class numbers of imaginary cyclic fields of degrees 4, 6, 8, and 10, Math. Comp. 61 (1993), no. 204, 881-887.

[4] D. R. Hayes, Explicit class field theory for rational function fields, Trans. Amer. Math. Soc. 189 (1974), 77-91.

[5] T. Metsänkylä, Bemerkungen über den ersten Faktor der Klassenzahl des Kreiskörpers, Ann. Univ. Turku. Ser. A I No. 105 (1967), 15 pp.

[6] M. Rosen, A note on the relative class number in function fields, Proc. Amer. Math. Soc. 125 (1997), no. 5, 1299-1303.

[7] _ Number Theory in Function Fields, Springer-Verlag, Berlin, 2002.

[8] D. Shiomi, A determinant formula for relative congruence zeta functions for cyclotomic function fields, J. Aust. Math. Soc. 89 (2010), no. 1, 133-144.

[9] K. Tateyama, Maillet's determinant, Sci. Papers College Gen. Ed. Univ. Tokyo 32 (1982), no. 2, 97-100.

[10] L. C. Washington, Introduction to Cyclotomic Fields, Springer-Verlag, New York, 1982.

Graduate School of Mathematics

NAGOYA UNIVERSITY

Chikusa-KU, NAGOYA 464-8602, JAPAN

E-mail address: m05019e@math.nagoya-u.ac.jp 